\title{
Daniel Proulx \\ La dualitude comme identité mystique chez Henry Corbin
}

\section{DUALITUDE AS MYSTICAL IDENTITY IN HENRY CORBIN'S THOUGHT}

Abstract: Henry Corbin is the creator of many neologisms. The term imaginal, related to the phrase mundus imaginalis, is probably the best known among them. Few researchers have come to realize that the term dualitude is also a Corbinian neologism. By first exploring the historical origin of the term in Corbinian thought, I propose then to explain the metaphysical role given by him to the term, but also the way in which the concept ensures the coherence of "mystical identity". As a matter of fact, Corbin places at the heart of "mystical identity" the principle of dualitude of the Self and the Ego. For Corbin, the metaphysical structure of monotheistic angelology rests on this principle and allows a personal relationship between the individual and the divine.

Keywords: Dualitude; Henry Corbin; Metaphysics; Active Intelligence; Angelology; Phenomenology.

\section{Daniel ProulX}

Université de Montréal, Montréal, Canada daniel.proulx2@gmail.com

DOI: 10.24193/cechinox.2021.41.04

\section{Ouverture}

Tenry Corbin est un philosophe-orien1 taliste né à Paris en 1903 et converti au protestantisme lorsqu'il était encore un jeune adulte $^{1}$. La date précise de sa conversion est inconnue, mais elle aurait eu lieu après 1922, alors qu'il quittait, après une année et sans raison apparente, le grand séminaire d'Issy-les-Moulineaux, et probablement avant 1927, date à laquelle on trouve un morceau de roman inachevé ${ }^{2}$, probablement partiellement autobiographique, où il est question du rejet du catholicisme ${ }^{3}$. Il est célèbre en philosophie notamment parce qu'il est le premier traducteur français de Heidegger ${ }^{4}$, mais aussi pour avoir été dans sa jeunesse une figure de proue de ceux que l'on nomme, suivant les travaux de Jean-Louis Loubet des Bayle ${ }^{5}$, les jeunes non-conformistes, et ce, en compagnie d'autres célèbres protestants comme Denis de Rougemont ${ }^{6}$. Corbin est un prodigieux intellectuel ayant une intarissable soif de connaissance ${ }^{7}$. Et quoique chacune des facettes de son intrigante personnalité nécessiteraient de multiples clarifications et approfondissements, la présente analyse met l'accent sur un néologisme corbinien qui a, pour le moment, moins attiré l'attention des chercheurs. Il s'agit du terme « dualitude». 
En effet, Corbin est surtout connu et reconnu pour avoir créé le néologisme « imaginal » auquel il a associé l'expression latine mundus imaginalis ${ }^{8}$, concept visant à traduire l'expression arabe álam al-mithāl, c'est-à-dire "monde imaginal » selon sa propre traduction. Un néologisme devenu courant chez les jungiens ${ }^{9}$ et chez les chercheurs qui s'intéressent à l'imaginaire ${ }^{10}$. Et, même si ce terme technique devrait lui aussi faire l'objet d'une réanalyse qui chercherait à distinguer l'approche de Corbin des récupérations contemporaines et de son sens dans la philosophie traditionnelle iranienne ; la présente analyse vise à mieux situer la structure fondamentale de la pensée corbinienne pour qu'ultérieurement soit possible un travail plus vaste d'histoire des idées des néologismes corbiniens.

Les lecteurs attentifs de l'œuvre de Henry Corbin se demandent certainement depuis la lecture du titre d'où est tirée l'expression "identité mystique ». Soyons clairs, l'expression ne se trouve pas dans l'œuvre de Henry Corbin. Il faut entendre cette expression comme un concept qui exprime un état dêtre relationnel, mais dont la relation ne se réduit ni à l'un ni à l'autre terme de l'alternative. Dans la pensée corbinienne, comme dans la pensée traditionnelle iranienne étudié par ce dernier, l'« identité mystique » s'exprime par la sentence mainte fois répétée : "Celui qui se connaît soi-même connaît son Seigneur ${ }^{11}$ ". Autrement dit, l'identité mystique se compose de la relation du moi et du soi. Ce que la mystique d'amour exprime plus poétiquement en disant : "Tu es moi (anta an $\hat{a})^{12}$ ». Une relation identitaire dialogique exprimée sans opposition ou assimilation et en dehors de la dualité et de l'unité.
Cette relation du moi et du soi, cet état non contradictoire de la relation du moi et du soi s'exprime par le concept de dualitude. Le terme explicite philosophiquement la relation non contradictoire du rapport anthropologique du moi et du $s o i^{13}$.

Quant au concept d'« identité mystique ", il doit se comprendre à l'aune d'une anthropologie du double sise dans une conception métaphysique spécifique. Dans la pensée corbinienne, l'identité est solidaire d'une anthropologie souvent exprimée en termes d'angélologie ou de mystique d'amour. Ainsi, chez Corbin, l'identité s'assimile à lêtre, elle n'est pas acquise ou découverte de l'extérieur. L'identité mystique ne s'ajoute pas à d'autres identités, il ne s'agit pas d'une catégorie additive. Ce texte ne traite pas de l'«identité $d u$ mystique ", mais bien de l'« identité mystique ». Connaître la mystique ne signifie pas devenir mystique ou devenir soi. Lidentité mystique est une identité ontologique, elle relève du dévoilement d'un état double, d'une syzygie, comme celle de l'homme et son ange ou encore de l'amant et l'aimé. Dans l'œuvre de Corbin, la philosophie est conçue comme exploration des conditions de possibilités intellectives de ce rapport caché, ésotérique ou intérieur et ce n'est qu'au prix de sa propre transformation intérieure que le philosophe acquière ce que l'on appelle l'identité mystique. Conséquemment le "vrai » philosophe est toujours présenté, par Corbin, comme un théosophe $e^{14}$.

Enfin, une dernière mise en garde est nécessaire, car l'expression de la mystique chez Corbin ne signifie jamais la fusion. Dans le corpus de textes qu'il a ressuscité et présenté au monde dans $s a$ "Bibliothèque iranienne $»^{15}$, le mystique ne fusionne 
jamais avec la divinité. Il y a plutôt chez ces auteurs un apophatisme fondamental qui empêche la fusion avec la divinité. Un apophatisme qui amène Corbin, à la fin de sa vie, à parler non plus de monothéisme, mais de théomonisme ${ }^{16}$, car le premier serait fusionnel, tandis que le second serait intégrateur de la différence sans la négation de l'alternative, seule posture métaphysique permettant de soutenir un « pluralisme métaphysique » ou une angélologie, sans que les anges soient des dieux, c'està-dire sans tomber dans le polythéisme. La mystique fusionnelle existe cependant, Hallāj et sainte Thérèse d'Ávila en sont des exemples ; dans l'œuvre corbinienne, elle est plutôt ontologiquement intégrative et s'assimile plus facilement à des auteurs de la tradition allemande comme Maître Eckhart ou Jakob Böhme, rapprochement régulièrement énoncé par Corbin lui-même.

\section{Origine et histoire du terme "dualitude "}

T a première occurrence du terme « duaMlitude " se trouve dans un texte publié en 1946, sous le titre Les motifs zoroastriens dans la philosophie de Sobrawardî17. Ce texte n'est certes pas l'œuvre de Corbin qui fit connaître le mot dans la francophonie, mais est certainement l'un des textes les plus significatifs pour Henry Corbin lui-même, car ce texte est celui de sa première conférence en terre iranienne. Cette conférence fut prononcée le $1^{\text {er }}$ novembre 1945 à Téhéran à l'invitation de la Société d'iranologie. Rappelons pour la chronologie que Henry et Stella Corbin habitaient à Istanbul pendant toute la Seconde Guerre mondiale. Le couple, marié en 1934, prend

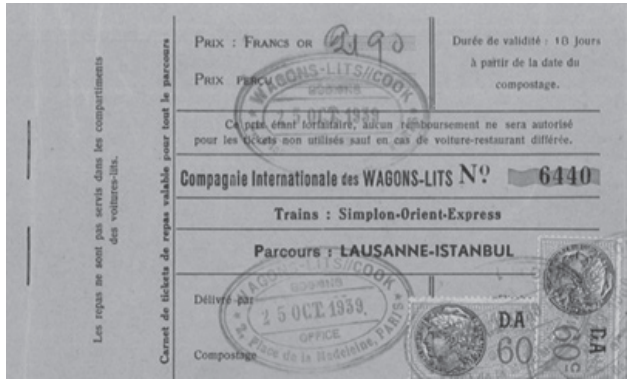

Figure 1. Billet de train pris par les Corbin, Archives Henry et Stella Corbin, École pratique des hautes études

le Simplon-Orient-Express le 25 octobre 1939. C'est le début de la grande aventure orientale des Corbin. Après la capitulation de l'Allemagne en mai 1945, les Corbin se préparent à se rendre en Iran. En effet, le « veilleur solitaire » de l'Institut français d'archéologie d'Istanbul s'était rendu en Turquie pour consulter les manuscrits de l'œuvre de Sohravardī qui avaient été signalés aux orientalistes de l'Europe par Hellmut Ritter ${ }^{18}$. De 1939 à 1945, il édite et traduit la totalité de l'œuvre de Sohra$\operatorname{vardi}^{19}$ à partir des manuscrits accessibles à Istanbul, mais il ne publie, à ce moment, qu'un volume d'œuvres ${ }^{20}$. Très précisément, le $1^{\text {er }}$ septembre 1945 les Corbin se mettent en route pour Téhéran. Environ 10 jours plus tard, ils arrivent en Iran et Henry démultiplie les prises de contact et les visites de fonds de manuscrits. C'est dans ce contexte que le $1^{\text {er }}$ novembre 1945 il prononce une conférence en français à la nouvelle de la Société d'iranologie ${ }^{21}$. Les statuts rédigés en anglais et en persan sont alors traduits en français par Corbin qui n'hésite pas à utiliser les termes " iranologie » et " iranologue »; il devient, également, sinon le créateur ${ }^{22}$, au moins le vecteur principal de la diffusion de ces termes dans la francophonie. 
Dans la conférence Les motifs zoroastriens dans la philosophie de Sobrawardî, on trouve certes la première occurrence du terme "dualitude ", mais cette conférence permet surtout de prendre conscience de la cohérence de sa pensée propre de sa prime jeunesse à sa mort, et ce, même si celle-ci se précise et évolue au fil des années. Par exemple, dans la note préliminaire qui précède le texte de la conférence, Corbin indique d'emblée son aversion de l'historicisme ${ }^{23}$ :

La philosophie de l'Ishrâq a été profondément ressentie par celui qui en parle, comme une philosophie de la Présence. Il eût été faux de la décomposer par les méthodes d'un historisme qui ne peut prendre le passé qu'en tant que passé, sans même être bien au clair sur les postulats qu'il implique. Malheureusement il nous était impossible de nous expliquer longuement ici sur les prémisses philosophiques de notre compréhension « au présent ». ${ }^{24}$

Une citation faisant parfaitement écho à un magnifique texte rédigé pour introduire la traduction anglaise des textes de la session Eranos de 1951 qui étaient consacrés au temps ${ }^{25}$. Rédigé un peu plus d'une dizaine d'années après Les motifs zoroastriens dans la philosophie de Sohrawardî, Corbin y adopte un style plus personnel qui implique, en toute vraisemblance, sa propre vision du monde ${ }^{26}$. Il rappelle que :

passé et futur, eux aussi, sont des attributs exprimés par des verbes; ils présupposent le sujet qui conjugue ces verbes, un sujet pour qui et par qui le seul temps existant est le présent, et chaque fois le présent. Dimensions du passé et du futur sont aussi bien chaque fois mesurées et conditionnées par la capacité du sujet qui les perçoit, par son instant. ${ }^{27}$

Une fois que l'on comprend que cette dimension au présent relève de la profonde attitude phénoménologico-herméneutique de Corbin qui cherche à circonscrire le principe métaphysique de la hiérarchie des êtres, on comprend mieux par ailleurs sa lecture de Sohravardī : et cela, car " [d] e l'illumination et du réfléchissement qui composent la relation entre la Lumière des Lumières et le Premier Archange, s'engendre une nouvelle Lumière : le second Archange. ${ }^{28} "$. De cette relation originelle émane la hiérarchie des êtres, une relation originelle qui est l'aurore de l'être de l'Amant et de l'Aimé. Une lumière de l'illumination orientale de l'être qui :

ordonne par couples la totalité des êtres de tous les univers en leur conférant les uns sur les autres une préséance semblable à celle de l'Aimé sur l'Amant - préséance qui fait ainsi se répéter la relation d'amour initial entre la Lumière des Lumières et le Premier archange dans la dualitude essentielle à tous les être, présents au "Matin » les uns des autres comme chaque être est présent à l'Ange ou Seigneur de son espèce. ${ }^{29}$

Ce que l'attitude au présent fait apparaître, c'est la structure duelle de tous les êtres, une structure duelle qui cohère l'être, qui ne le divise pas. Voilà pourquoi le terme "dualitude » exprime la cohérence duelle de lêtre. 
Le concept sera ensuite progressivement développé dans d'autres ouvrages. Il le rend de plus en plus autoportant et l'applique plus largement à une structure métaphysique qu'il retrouve à l'extérieur de l'œuvre de Sohravardī. Dans Avicenne et récit visionnaire, composé une dizaine d'années plus tard, Corbin clarifie parfaitement les symboles de l'Étranger et du Retour :

[a]u moment où l'âme se découvre comme étrangère et solitaire dans un monde qui lui avait été familier, se profile à son horizon une figure personnelle, s'annonçant personnellement à elle, parce qu'elle symbolise avec son fond le plus intime. Autrement dit, l'âme se découvre comme étant la contrepartie terrestre d'un autre être avec lequel elle forme une totalité de structure duelle. Les deux éléments de cette dualitude peuvent être désignés comme le Soi et le Moi ou encore comme le Moi céleste transcendant et le Moi terrestre. ${ }^{30}$

\section{Métaphysique de la dualitude}

$\mathrm{P}$ our contextualiser la notion de « dualitude » et pour comprendre par-delà le positivisme et le dualisme omniprésent au $\mathrm{XIX}^{\mathrm{e}}$ et au $\mathrm{XX}^{\mathrm{e}}$ siècles, il faut, du point de vue de Corbin, remonter à la philosophie médiévale afin de circonscrire les raisons profondes qui rendent l'« esprit occidental » impropre à saisir des notions tierces inclusives ou contradictorielles, pour reprendre des concepts phares de Stéphane Lupasco $^{31}$. Pour Corbin, les difficultés conceptuelles liées à la notion de dualitude découleraient de la perte de l'intelligence agente, advenue selon l'iranologue, lors des confrontations philosophiques autour de la pensée d'Averroès au XIV ${ }^{\mathrm{e}}$ siècle.

L'histoire de la philosophie corbinienne se confond dans son œuvre avec l'histoire de la séparation de la foi et de la raison, car si l'histoire de la philosophie et l'histoire de la spiritualité sont inséparables dans le monde islamique, en Occident les choses se passent autrement. Pour Corbin le nœud du problème de l'occidentalisme doit être interrogé via l'étude des causes de cette séparation de la «spiritualité et la philosophie " ou plus précisément de la séparation entre « la prophétie et la philosophie ", surtout dans le contexte des religions du Livre. Pour lui, la cause de cette séparation est la diffusion de l'averroïsme en Europe ou plutôt la réaction occidentale à la philosophie du Maître de Cordoue. Pour Corbin, la philosophie aurait perdu sa relation à la sophia à ce moment. Corbin l'exprime sans détour dans l'Histoire de la philosophie islamique ${ }^{32}$.

Cette hypothèse originale de Corbin pour expliquer l'occidentalisme, c'est-àdire la pensée réductrice et historicisante, est pour lui associée à Averroès et il en fait le symbole du divorce de l'Occident et l'Orient en insistant sur l'exil oriental d'Ibn 'Arabī'33 qui débute en 1198, année de la mort d'Averroès. Corbin a développé en détail cette hypothèse dans la longue introduction ajoutée aux deux conférences prononcées au Cercle Eranos en 1955 et 1956, textes recueillis dans L'imagination créatrice dans le soufisme d'Ibn 'Arabì. L'exil oriental d'Ibn 'Arabī est alors considéré comme une " catastrophe métaphysique ${ }^{34}$ " pour l'Occident, car cela aurait réduit à néant, d'après Corbin, l'accès au monde des Âmes célestes, des Anima calestes. L'organe nécessaire pour accéder au monde des anges et de 
l'âme aurait été amputé à l'Occident dans la confrontation philosophique avec la pensée d'Averroès. Une confrontation qui a opposé, au XIII ${ }^{\mathrm{e}}$ siècle, les facultés de philosophie et de théologie de l'Université de Paris ${ }^{35}$. Ces disputationes aurait eu comme conséquence de verrouiller hermétiquement les champs philosophique et théologique, créant ainsi la première véritable « discontinuité » disciplinaire. Parce que, selon Corbin, la philosophie et la théologie sont deux facettes d'une même médaille, " tout antagonisme entre théologie et philosophie est a priori surmonté en une hikmat ilāhìya, une "sagesse divine" 36 "; celle-ci impossible sans une intelligence agente qui, à l'image de la dualitude humaine du Moi terrestre et du Moi céleste $^{37}$, doit être simultanément "Ange de la Révélation et de la connaissance ${ }^{38}$ ». Pour Corbin, la séparation radicale du théologique et du philosophique aurait alors à se souvenir de sa théo-sophie spécifique pour surmonter la séparation. Une réconciliation qui soutient en profondeur la notion de philosophie islamique ${ }^{39}$.

Dans la conception de l'histoire de Corbin, ces transformations auraient entraîné la perte de l'intelligence agente et permis l'émergence du rationalisme historiciste. Une vision du monde qui aurait empêché une imagination de second degré, l'imaginal, c'est-à-dire une imagination fondée et produite "dans » et "à partir » de l'imaginal, par opposition à une phantaisie qui, en déformant et s'inspirant du réel empirique produit du chimérique, de l'imaginaire, de l'irréel ${ }^{40}$. Le regard « oriental » de Corbin ferait ainsi apparaître une déficience de la compréhension " occidentale » de l'imaginaire. Et c'est cette idée qui liera Henry Corbin et Gilbert Durand. Cette réappropriation est importante, car si la pensée corbinienne n'a pas fait " école », c'est tout le contraire de celle de Durand qui a fondé, à Grenoble, en 1966, le premier Centre de recherche sur l'imaginaire (CRI). Et parce qu'il s'inspire abondamment de l'œuvre de Corbin ${ }^{41}$, celui-ci s'est retrouvé parachuté dans la constellation de l'imaginaire ${ }^{42}$. Le premier terreau de réception propice à l'implantation de la conception corbinienne de l'imagination et de l'histoire est donc le réseau des Centres de Recherche sur l'Imaginaire.

Cette hypothèse corbinienne de la perte de l'intelligence agente, reprise et amplifiée par Gilbert Durand ${ }^{43}$, n'a jamais vraiment été confrontée, décortiquée ou vérifiée. La perte de l'intelligence agente serait la cause de la « catastrophe métaphysique » et du début de l'historicisme comme « socialité envahissante ${ }^{44}$ ", car sans elle, le monde intermédiaire - le mundus imaginalis - n'est pas possible, aux yeux de Corbin. L'hypothèse est centrale, on peut presque dire que toute son œuvre est construite comme une exploration de l'espace apparitionnel du Deus absconditus, du Dieu caché. Une apparition du Deus absconditus qui n'a de sens que si elle s'opère en dualitude. La problématique est déjà présente dans ses écrits " théologiques » de jeunesse ${ }^{45}$, avant de faire l'objet d'un premier développement dans Avicenne et le récit visionnaire.

En quatre pages d'une incroyable densité $^{46}$, Corbin explique que la théorie des Intelligences hiérarchiques, héritée de Fārābī, et comprise comme une angélologie, fonde à la fois l'anthropologie et la cosmologie. La Création, dans ce contexte, consiste «dans l'acte même de la pensée divine se pensant soi-même ${ }^{47}$ "; cette pensée est la Première émanation, la Première intelligence. Dans une série d'actes instaurateurs, 
où les Intelligences contemplent leur Principe nécessaire, apparait la double hiérarchie des Dix intelligences chérubiniques et de leur Âme céleste. Chaque Intelligence fait un acte de contemplation triple ; d'abord " elle contemple le principe qui la nécessite dans lêtre ", ce qui génère l'Âme motrice du premier Ciel, la Sphère des Sphères. Ensuite, d'émanation en émanation, la Dixième intelligence «n'a plus la force de produire à son tour une autre Âme unique ». Enfin, c'est l'explosion de l'Émanation, la part d'ombre restante forme alors le monde sublunaire ; la Dixième intelligence est ainsi comprise comme Intelligence agente d'où découlent les âmes humaines, le Dator formarum ou le Donneur de forme. On y retrouve une conception très différente de celle de Thémistius ou de saint Thomas qui n'acceptent pas que l'Intelligence agente soit extrinsèque à l'humain. Mais, à la différence d'Alexandre d'Aphrodise, l'Intelligence agente n'est pas identifiée à Dieu. Une conception également différente de celle d'Averroès par qui l'idée de «création " vendra poindre à travers le système émanentiste d'Avicenne. De plus, le " monopsychisme » averroïste ne niait pas l'Intelligence agente séparée, mais réduisait l'intelligence humaine à une simple " complexion organique » qui ne laissait aucune place à la survie de l'âme individuelle. Il est à noter que pour Corbin la survie post mortem de l'âme semble une nécessité à sa propre conception du monde. À la fin du chapitre sur Averroès et l'averroïsme, dans lequel il ne parle pas d'Averroès, mais seulement de la manière dont sa théorie s'oppose à celle d'Avicenne et des ishräqüyün, il note : «Tout ce que l'on peut dire, c'est qu'il y a de l'éternité dans l'individu, mais ce qu'il y a d"éternisable" en lui appartient totalement à la seule Intelligence agente, non pas à l'individu. ${ }^{48}$ " Dans la dualitude essentielle à tous les êtres, «l'âme se découvre comme étant la contrepartie terrestre d'un autre être avec lequel elle forme une totalité de structure duelle. ${ }^{49}$ " Dualitude du soi et du moi, du céleste et du terrestre, ou encore dimension polaire de l'homme et de son ange $\mathrm{e}^{50}$.

Sans dualitude, c'est la catastrophe métaphysique, car l'humain ne serait plus en mesure de réintégrer la hiérarchie des êtres célestes ; l'humain serait alors esseulé, exilé s'il n'est plus en mesure de penser et d'intégrer la dualitude.

Sans dualitude, il n'y a plus de symbole, seulement des signes.

Sans dualitude, il n'y a plus d'imagination vraie, d'imaginal, il ne reste que la phantasia.

Sans dualitude, il n'y a plus de sophia, seulement la ratio ${ }^{51}$.

\section{Actualité de la dualitude}

Corbin a mis en lumière, dans les récits métaphysiques d'Avicenne ${ }^{52}$ et de Sohravardi ${ }^{53}$, l'illumination orientale de lêtre ; voulant dépasser les simples descriptions historicistes et mécanistes de ces récits et afin d'en rendre la portée intérieure et herméneutique il a systématiquement repositionné phénoménologiquement le cadre logique et temporel des symboles mis au présent par les philosophes, théosophes et mystiques ; ces symboles, il les a ressuscités des méandres de la mémoire d'histoire. Ainsi, Corbin a légitimé et rendu opérationnelle une certaine approche du fait religieux et du symbole qui a ouvert la voie à plusieurs autres chercheurs. L'un des pas les plus importants dans cette récupération élargie de la pensée corbinienne de la dualitude a été fait par Gilbert Durand. En effet, entre la publication 
des Structures anthropologiques de l'imaginaire en 1960 et la publication de L'imagination symbolique en 1964, on observe le choc de la rencontre de la pensée corbinienne; ce choc rendu visible par le passage du vocabulaire influencé par l'ethnologie, l'anthropologie et la psychanalyse à des vocables plus métaphysiques et religieux. Par exemple, citant L'imagination créatrice dans le soufisme d'Ibn 'Arabì, Durand conclut que : «Le symbole est donc une représentation qui fait apparaître un sens secret, il est l'épiphanie d'un mystère. ${ }^{54}$ " La dimension symbolique est une dimension instauratrice pouvant chaque fois donner au présent une nouvelle dimension; celle-ci est un processus qui fait apparaître des dimensions cachées, ésotériques de l'être, car comme le précise Corbin, un symbole n'est jamais expliqué une fois pour toutes, mais il est toujours à déchiffrer ${ }^{55}$ comme une partition musicale qui appelle chaque fois une nouvelle exécution qui ne sera jamais identique à la précédente et qui s'exécute toujours au présent ${ }^{56}$.

Cette requalification du symbole pardelà le signe, doit se faire par-delà la dualité ou par-delà le cartésianisme. C'est ce que Corbin a retrouvé dans la philosophie iranienne, par-delà le thomisme et la perte de l'angélologie et c'est ce que Durand retrouve par-delà Descartes et les iconoclastes.

Enfin, partant du champ philosophique de la dualitude bien établi par Jean-Jacques Wunenburger dans La raison contradictoire ${ }^{57}$, il y aurait lieu de retrouver la dualitude en montrant que le principe épistémologique qui est au cœur du terme fut exploré par Stéphane Lupasco, par Carl Gustav Jung, par Gilbert Durand, par Paul Ricœur, par Edgar Morin ou par Basarab Nicolescu. Une requalification logique qui permettrait de relire les textes anciens non seulement sous un mode analogique, mais aussi dans une dynamique complexifiante. Une attitude philosophique où sous la lunette de la dualitude, les différents "-ismes " (capitalisme, fascisme, intégrisme, etc.) s'imposeraient moins à l'esprit comme des absolus.

Navigant entre les écueils de l'historicisme et du subjectivisme, Corbin cherchait à établir une méthode ou plus précisément une « approche » qui ne réduit pas à néant le contenu métaphysique de ce qu'il cherchait à comprendre ${ }^{58}$. À cet égard, il se dit phénoménologue, mais sans s'attacher à une école phénoménologique particulière, car Corbin nest pas un commentateur analytique de la phénoménologie; il ne veut pas faire de la phénoménologie une technique philosophique, toute sa connaissance philosophique de la phénoménologie étant imbriquée dans une anthropologie visant à rendre compte au présent de ce qui a été vu et vécu. Pour Corbin, la phénoménologie signifie rencontrer les phénomènes " là où ils ont lieu et où ils ont leur lieu. ${ }^{59}$ " Il s'agit pour lui « de rencontrer le fait religieux en laissant se montrer l'objet religieux tel qu'il se montre à ceux à qui il se montre. ${ }^{60}{ }$ Corbin, qui était aussi un excellent musicien ${ }^{61}$, utilise ici une métaphore musicale pour rendre compte de la difficulté à cerner le lieu du phénomène religieux. La phénoménologie serait comme de « cerner le monde qui se révèle à nous par les rythmes de l'espace sonore ${ }^{62}$ "?

\section{Ouverture conclusive}

Dans La raison contradictoire, Jean-
Jacques Wunenburger sétait donné
comme projet de [s] tatuer sur les voies qui permettraient de renouer avec un entrecroisement 
inextricable de l'identité et de l'altérité, de l'unité et de la pluralité, de la répétition et du changement, [ce qui] devrait permettre de retrouver un gyroscope intellectuel, capable d'orienter l'homme au milieu d'une crise profonde de ses référentiels. ${ }^{63}$
Un projet intellectuel éclairé et précédé par l'ensemble des travaux philosophiques de Henry Corbin sur le temps, la phénoménologie et l'herméneutique et qui participe aujourd'hui à soutenir la réflexion au cœur des centres de recherches sur l'imaginaire.

\section{BibLIOGRAPHIE}

Camilleri, Sylvain et Proulx, Daniel, « Martin Heidegger et Henry Corbin : lettres et documents (19301941)», dans Bulletin heideggérien, vol. 4, 2014, p. 4-63.

Corbin, Henry, Avicenne et le récit visionnaire, Lagrasse, Verdier, coll. « Islam spirituel », 1999.

Corbin, Henry, Corps spirituel et Terre céleste, de l'Iran mazdéen à l'Iran shî̀ite, $3^{\mathrm{e} e ́ d ., ~ P a r i s, ~ B u c h e t / C h a s-~}$ tel, coll. « La Barque du soleil », 2005 [1960].

Corbin, Henry, « De la théologie apophatique comme antidote du nihilisme », dans L'Impact planétaire de la pensée occidentale rend-il possible un dialogue réel entre les civilisations? édité par Centre iranien pour l'étude des civilisations, Paris, Berg International, 1979, p. 33-72.

Corbin, Henry, En islam iranien, aspects spirituels et philosophiques - Le shî̀isme duodécimain, vol. 1, Paris, Gallimard, coll. « Tel» n $189,1971$.

Corbin, Henry, Histoire de la philosophie islamique, Paris, Gallimard, coll. « folio/essais » n 39, 1986.

Corbin, Henry, L’homme de lumière dans le soufisme iranien, Chambéry, Présence, coll. « Le Soleil dans le cœur » $n^{\circ} 4,1971$.

Corbin, Henry, L'imagination créatrice dans le soufisme d'Ibn 'Arabī, Paris, Entrelacs, 2006 [1958].

Corbin, Henry, "Le temps d'Eranos », dans Roger Godel de l'bumanisme à l'bumain, Paris, Les Belles Lettres, 1963, p. 177-186.

Corbin, Henry, Les motifs zoroastriens dans la philosophie de Sobrawardì Shaykb-ol-Ishrâq (ob. 587/1191), préf. de Pouré Davoud, Tehéran, éditions du courrier, coll. « Publications de la Société d'iranologie » $n^{\circ} 3,1946$.

Corbin, Henry, « Note sur Existence et Foi », dans Hic et Nunc, n 6, juillet 1933, p. 51-57.

Corbin, Henry, "The Time of Eranos ", trad. par Ralph Manheim, dans Man and time. Papers from the Eranos Yearbooks édité par Joseph Campbell, London, Princeton University Press, coll. « Bollingen Series » n 30/3, 1983 [1957], p. XIII-XX.

Corbin, Henry, Roger Jézéquel, Roland de Pury, et al., « Manifeste », dans Hic et Nunc, nº 1, novembre 1932, p. 1-3.

Durand, Gilbert, « Henry Corbin entre l'Orient et l'Occident », dans Les nouvelles littéraires, vol. 56, ${ }^{\circ}$ 2657, 26 octobre 1978, p. 8.

Durand, Gilbert, «Hommage à Henry Corbin - La pensée d'Henry Corbin et le temple maçonnique », dans Temple et contemplation, Paris, Entrelacs, 2006, p. 9-21.

Durand, Gilbert, «Homo proximi orientis: science de l'homme et islam spirituel », dans Science de l'homme et tradition, Paris, Albin Michel, 1996 [1979], p. 91-139.

Durand, Gilbert, L'imagination symbolique, 5e éd., Paris, P.U.F., coll. « Quadrige » n 51, 2008 [1964].

Durand, Gilbert, «La reconquête de l'Imaginal », dans Henry Corbin édité par Christian Jambet, Paris, L'Herne, coll. «Cahiers de l'Herne » n³9,1981, p. 266-273.

Durand, Gilbert, " Psychanalyse de la neige », dans Mercure de France, vol. 318, mai-août 1953, p. 615-639.

Durand, Gilbert, «Tâches de l'Esprit et impératif de l'Être », dans Eranos-Jabrbuch, vol. XXXIV/1965, 1966, p. 303-360. 
Heidegger, Martin, Qu'est-ce que la métaphysique? : suivi d'extraits sur l'être et le temps et d'une conférence sur Hölderlin, trad. par Henry Corbin, Paris, Gallimard, 1938.

Heidegger, Martin, «Qu'est-ce que la métaphysique ? Leçon inaugurale donnée à l'Université de Fribourg-en-Brisgau le 24 juillet 1929 », trad. par Henry Corbin, dans Bifur, vol. 8, 1931, p. 9-27.

Jambet, Christian, Quest-ce que la philosophie islamique?, Paris, Gallimard, coll. « folio/essais » $\mathrm{n}^{\circ}$ 547, 2011.

Loubet del Bayle, Jean-Louis, Les non-conformistes des années 30 : une tentative de renouvellement de la pensée politique française, éd. rév. et actualisée, Paris, du Seuil, coll. « Points. Histoire » n 295, 2001 [1969].

Nicolescu, Basarab, Qu'est-ce que la réalité ? Réflexions autour de l'œuvre de Stéphane Lupasco, Montréal, Liber, 2009.

Piché, David et Lafleur, Claude, La condamnation parisienne de 1277, Paris, Vrin, 1999.

Proulx, Daniel, Catalogue du Fonds Henry et Stella Corbin, conservé par le Grand équipement documentaire du Campus Condorcet, École pratique des hautes études, Paris, 2016, EPHE BSR 5COR, http://www.calames.abes.fr/pub/\#details?id=FileId-2016.

Proulx, Daniel, « La connaissance transdisciplinaire et le sacré », dans Expliquer, comprendre et débattre autour du religieux. Neutralité ou engagement ? édité par Bernard Gagnon, Daniel Proulx et Samia Amor, Québec, Presses de l'Université Laval, 2020, p. 31-46.

Proulx, Daniel, «La figure de la sophia chez Henry Corbin », dans Femme, Erôs et Philosophie édité par Elen-Dania Diotte-Besnou, Daniel Proulx et Jean-Michel Counet, Louvain-la-Neuve, EME, coll. «Religions des philosophes » n 5, 2016, p. 289-317.

Proulx, Daniel, « La non-dualité chez Henry Corbin », dans La non-dualité, perspectives philosophiques, scientifiques, spirituelles édité par Jean-Michel Counet, Louvain-la-Neuve, Peeters, coll. « Bibliothèque philosophique de Louvain » $n^{\circ} 112,2021$, p. 103-123.

Proulx, Daniel, « La perception auditive comme perception spirituelle chez Henry Corbin », dans Quaderni di Studi Indo-mediterranei - Oikosophia édité par Daniela Boccassini, Milan, Mimesis n¹0, 2017, p. 227-246.

Proulx, Daniel, «Les racines “imaginaires” de la transdisciplinarité », dans L'imaginaire durandien Enracinements et envols en Terre d'Amérique édité par Raymond Laprée et Christian Bellehumeur, Québec, Presses de l'Université Laval, 2013, p. 169-181.

Pury (de), Roland, Lettres d'Europe un jeune intellectuel dans l'entre-deux-guerres, 1931-1934, Genève/ Paris, Labor et fides/du Cerf, 2010.

Ritter, Hellmut, « Philologika IX », dans Der Islam: Zeitschrift für Geschichte und Kultur des Islamischen Orients, vol. 24, 1937, p. 270-286.

Sohravardī, Shihāboddīn Yahyā, L'archange empourpré, quinze traités et récits mystiques, éd. et trad. de l'arabe et du persan, intro. et notes de Henry Corbin, Paris, Fayard, coll. «L'espace intérieur » n 14, 1976.

Suhrawardī, Shihâb al-Dîn Yahyà, Opera metaphysica et mystica, edidit et prolegomenis instruxit Henricus Corbin, vol. 1, Istanbul, Maarif Matbaasi, coll. « Bibliotheca islamica » nº 16a, 1945.

Unesco (éd.), La science face aux confins de la connaissance : la déclaration de Venise : colloque international, Paris, du Félin, 1987.

Wunenburger, Jean-Jacques, La raison contradictoire, Paris, Albin Michel, 1990.

\section{NoTES}

1. À noter que Corbin ne se convertira jamais à l'islam et demeurera protestant jusqu'à sa mort.

2. Les textes du Fonds Henry et Stella Corbin sont très fragmentaires, mais les documents donnent à penser qu'il s'agissait d'une ébauche de roman.

3. Ce « roman inachevé» est conservé dans le Fonds Henry et Stella Corbin de l'École pratique des hautes études. Daniel Proulx, Catalogue du Fonds Henry et Stella Corbin, conservé par le Grand équipement 
documentaire du Campus Condorcet, École pratique des hautes études, Paris, 2016, EPHE BSR 5COR, 48 dimabs, 16 mètres linéaires, http://www.calames.abes.fr/pub/\#details?id=FileId-2016.

4. D'abord la traduction d'un premier texte de Heidegger publié en 1931 dans la revue Bifur, mais cette traduction a été honnis par Corbin lui-même dans sa bibliographie, préférant insister sur le recueil de traductions publié en 1938. Martin Heidegger, «Qu’est-ce que la métaphysique ? Leçon inaugurale donnée à l'Université de Fribourg-en-Brisgau le 24 juillet 1929 », trad. par Henry Corbin, dans Bifur, vol. 8, 1931, p. 9-27. Martin Heidegger, Quest-ce que la métaphysique? : suivi d'extraits sur l'être et le temps et d'une conférence sur Hölderlin, trad. par Henry Corbin, Paris, Gallimard, 1938. Sur Corbin et Heidegger, voir Sylvain Camilleri et Daniel Proulx, "Martin Heidegger et Henry Corbin : lettres et documents (1930-1941) », dans Bulletin heideggérien, vol. 4, 2014, p. 4-63.

5. Jean-Louis Loubet del Bayle, Les non-conformistes des années 30 : une tentative de renouvellement de la pensée politique française, éd. rév. et actualisée, Paris, du Seuil, 2001 [1969].

6. Voir par exemple les articles de Corbin publiés dans la revue Hic et Nunc dont il est l'un des cofondateurs, et plus précisément le manifeste fondateur de la revue. Henry Corbin, Roger Jézéquel, Roland de Pury, Denis de Rougemont et Albert-Marie Schmidt, " Manifeste », dans Hic et Nunc, $\mathrm{n}^{\circ}$ 1, novembre 1932, p. 1-3.

7. « Ce Corbin est bien l'homme le plus savant que j’aie jamais rencontré. Il est étouffant. Il sait le français, l'allemand, l'italien, l'espagnol, l'anglais, l'arabe et le persan. Et assez pour lire : le sanskrit, le turc, le hollandais, le suédois et le latin. Il est plongé dans la mystique arabe et n'ignore pas un recoin de la philosophie et de la théologie allemande contemporaine, dont il connaît tous les coryphées personnellement. Mais surtout, il n'est rien de tout ce qu'il sait, qui ne soit pour lui en rapport direct avec sa tâche immédiate, rien qui ne soit existentiel, c'est-à-dire qu'il hait l'histoire lorsqu'elle est autre chose qu'une "présentation" des choses et des hommes, et s'indigne avec enthousiasme contre tant de méthodes françaises psychologiques, précautionneuses et irréelles. Simplement dit, il est chrétien. C'est un type de Français assez rare et bienfaisant. » Roland de Pury, Lettres d'Europe - Un jeune intellectuel dans l'entre-deux-guerres, 1931-1934, Genève/Paris, Labor et fides/du Cerf, 2010, p. 188-189.

8. À noter que l'expression latine mundus imaginalis ne se retrouve pas dans les traités médiévaux. Cette expression latine est une autre création corbinienne.

9. Notamment par la récupération du terme par James Hillman que Corbin a bien connu. Ils se sont côtoyés au Cercle Eranos pendant de nombreuses années.

10. Cette récupération passe essentiellement par les centres de recherche sur l'imaginaire (CRI) parce que Gilbert Durand, grand ami de Corbin et très proche de sa pensée, est le fondateur et l'animateur de l'ancêtre de tous les CRI : le CRI de Grenoble.

11. Cette connaissance métaphysique de son seigneur, c'est l'objet du voyage en Orient ou plus précisément du voyage dans l'Orient de l'être. Voir par exemple, Henry Corbin, En islam iranien, aspects spirituels et philosophiques - Le shî̀isme duodécimain, vol. 1, Paris, Gallimard, 1971, p. 286-290.

12. Henry Corbin, L’homme de lumière dans le soufisme iranien, Chambéry, Présence, 1971, p. 23.

13. Sur le développement de cette idée dans l’œuvre de Martin Buber, voir Daniel Proulx, «La connaissance transdisciplinaire et le sacré ", dans Expliquer, comprendre et débattre autour du religieux. Neutralité ou engagement?, sous la direction de Bernard Gagnon, Daniel Proulx et Samia Amor, Québec, Presses de l'Université Laval, 2020, p. 42-44.

14. Un terme qui doit être compris étymologiquement selon la mise en garde mainte fois répétée par Corbin.

15. À sa mort, la « Bibliothèque iranienne » qu'il dirigeait comptait 22 volumes. Voir la liste complète des volumes : https://ifriran.org/publications/bibliotheque-iranienne-bi/.

16. Henry Corbin, « De la théologie apophatique comme antidote du nihilisme », dans L'Impact planétaire de la pensée occidentale rend-il possible un dialogue réel entre les civilisations?, sous la direction de Centre iranien pour l'étude des civilisations, Paris, Berg International, 1979, p. 33-72.

17. Henry Corbin, Les motifs zoroastriens dans la philosophie de Sohrawardî Shaykh-ol-Ishrâq (ob. 587/1191), préf. de Pouré Davoud, Tehéran, éditions du courrier, 1946, p. 31. 
18. Hellmut Ritter, «Philologika IX », dans Der Islam: Zeitschrift für Geschichte und Kultur des Islamischen Orients, vol. 24, 1937, p. 270-286.

19. Ziai et Walbridge, qui ont repris le travail d'édition de l'œuvre de Sohravardī, là où Corbin et Nasr l'avaient laissé, affirment que Corbin ne s'est jamais intéressé à la partie de l'œuvre de ce dernier consacrée à la logique, privilégiant les parties métaphysiques et mystiques. Or, Corbin a traduit la totalité de l'œuvre de Sohravardì lorsqu'il était à Istanbul. Cependant, pour une raison inconnue, il n'a jamais publié ou retravaillé ces traductions qui sont maintenant dormantes dans le Fonds Henry et Stella Corbin de l'École pratique des hautes études de Paris.

20. Shihâb al-Dîn Yahyyà al- Suhrawardī, Opera metaphysica et mystica, edidit et prolegomenis instruxit Henricus Corbin, vol. 1, Istanbul, Maarif Matbaasi, 1945, LXXXI, 511 p.

21. Corbin a participé à la fondation de la Société d'iranologie.

22. Cet usage lui vient peut-être de sa vaste connaissance de la langue allemande ou encore peut-être est-ce une influence de son maître Louis Massignon, car le terme iranologie apparaît dans le $12^{\mathrm{e}}$ volume de la Revue des études islamiques, dirigée par Massignon, en 1938.

23. Le terme utilisé dans le texte est « historisme ». Voir supra la note 6 qui précise que cette aversion de l'historicisme est encore plus ancienne.

24. Henry Corbin, Les motifs zoroastriens dans la philosophie de Sobrawardî, p. 8.

25. Henry Corbin, «The Time of Eranos ", trad. par Ralph Manheim, dans Man and time. Papers from the Eranos Yearbooks, sous la direction de Joseph Campbell, London, Princeton University Press, 1983 [1957], p. XIII-XX.

26. À noter que le « je » corbinien est rarissime dans son œuvre. Ce style d'écriture rend difficile l'analyse et la séparation de ce qui appartient en propre à la pensée corbinienne de ce que textes qu'il analyse affirment.

27. Henry Corbin, «Le temps d'Eranos », dans Roger Godel de l'bumanisme à l'bumain, Paris, Les Belles Lettres, 1963, p. 182.

28. Henry Corbin, Les motifs zoroastriens dans la philosophie de Sohrawardi, p. 30.

29. Ibid., p. 31.

30. Henry Corbin, Avicenne et le récit visionnaire, Lagrasse, Verdier, 1999, p. 32.

31. Sur l'œuvre de Stéphane Lupasco, voir Basarab Nicolescu, Qu'est-ce que la réalité? Réflexions autour de l'cuvre de Stéphane Lupasco, Montréal, Liber, 2009. À noter que Corbin a des relations avec plusieurs roumains expatriés, notamment Mircea Eliade et Emil Cioran, qui était par ailleurs son voisin à Paris rue de l'Odéon. Il est possible qu'il ait rencontré ou entendu parlé de l'œuvre de Lupasco.

32. «Tandis que l'avicennisme, en Occident pour une courte durée, en Iran jusqu'à nos jours, tendait à fructifier en vie mystique, l'averroïsme latin aboutissait à l'averroïsme politique de Jean de Jandun et de Marsile de Padoue (XIV siècle). De ce point de vue, les noms d'Avicenne et d'Averroës pourraient être pris comme les symboles des destinées spirituelles respectives qui attendaient l'Orient et l'Occident, sans que la divergence de celles-ci soit imputable au seul averroïsme. On a vu comment Abû'l-Barakât Baghdâdî poussait à la limite la gnoséologie avicennienne, en reconnaissant pour chaque individu, ou tout au moins pour les individus composant une même famille spirituelle, une Intelligence agente distincte, qui est une entité spirituelle "séparée". Les solutions données au problème de l'Intelligence agente, nous l'avons déjà relevé, sont hautement significatives. Lorsque saint Thomas d'Aquin, par exemple, accorde à chaque individu un intellect agent, mais sans que cet intellect soit une entité spirituelle "séparée", du même coup se trouve brisée la relation immédiate de l'individu avec le monde divin, telle qu'elle était fondée par la doctrine avicennienne de l'Intelligence agente, celle-ci identifiée avec l'Esprit-Saint ou l'Ange de la Révélation. Une fois brisée cette relation qui, sans intermédiaire terrestre, établissait l'autonomie de l'individualité spirituelle, l'autorité de l'Église se substitue à la norme personnelle de Hayy ibn Yaqzân. Au lieu que la norme religieuse, en tant qu'initiation essentiellement individuelle, signifiât liberté, c'est désormais contre elle, parce que socialisée, que se dresseront les insurrections de l'esprit et de l'âme. Il arrivera que cette norme, une fois socialisée, cesse d'être religieuse, vire du monothéisme au monisme, de l'idée d'Incarnation 
divine à celle d'Incarnation sociale. Alors ici surtout, il faut être attentif aux différences. » Henry Corbin, Histoire de la philosophie islamique, Paris, Gallimard, 1986, p. 344-345.

33. Gilbert Durand écrit : "Ibn 'Arabī écœuré, en tout cas lassé, part vers l'Orient des lumières. " Unesco (éd.), La science face aux confins de la connaissance : la déclaration de Venise : colloque international, Paris, du Félin, 1987, p. 108.

34. L'expression « catastrophe métaphysique » appartient à Corbin. L'idée est déjà présente en 1954 dans Avicenne et le récit visionnaire; elle est ensuite développée dans l'introduction ajoutée à l'Imagination créatrice dans le soufisme d'Ibn 'Arabì en 1958. Henry Corbin, Avicenne et le récit visionnaire, p. 29-34 et $130-156$.

35. À noter que les condamnations d'Étienne Tempier contre les averroïstes de Paris en 1270 et 1277 est un événement marquant de cette histoire de la réception d'Averroès en Occident. David Piché et Claude Lafleur, La condamnation parisienne de 1277, Paris, Vrin, 1999.

36. Henry Corbin, En islam iranien, vol. 1, p. 46.

37. Henry Corbin, Avicenne et le récit visionnaire, p. 32.

38. Henry Corbin, L'imagination créatrice dans le soufisme d'Ibn 'Arabì, Paris, Entrelacs, 2006 [1958], p. 42. Pour aller plus loin dans la conception métaphysique de la dualitude dans la pensée corbinienne voir, Daniel Proulx, «La non-dualité chez Henry Corbin », dans La non-dualité, perspectives philosophiques, scientifiques, spirituelles édité par Jean-Michel Counet, Louvain-la-Neuve, Peeters, coll. « Bibliothèque philosophique de Louvain » $\mathrm{n}^{\circ}$ 112, 2021, p. 103-123.

39. Sur cette notion, voir Christian Jambet, Quiest-ce que la philosophie islamique?, Paris, Gallimard, 2011.

40. Sur cette définition de l'imagination comme imaginatio vera: Henry Corbin, Corps spirituel et Terre céleste, de l'Iran mazdéen à l'Iran shîite, $3^{\mathrm{e}}$ éd., Paris, Buchet/Chastel, 2005 [1960], p. 102-106.

41. Gilbert Durand, «Henry Corbin entre l'Orient et l'Occident ", dans Les nouvelles littéraires, vol. 56, $\mathrm{n}^{\circ}$ 2657, 26 octobre 1978, p. 8. Gilbert Durand, « Homo proximi orientis : science de l'homme et islam spirituel ", dans Science de l'homme et tradition, Paris, Albin Michel, 1996 [1979], p. 91-139. Gilbert Durand, « La reconquête de l'Imaginal », dans Henry Corbin, sous la direction de Christian Jambet, Paris, L'Herne, 1981, p. 266-273. Gilbert Durand, « Hommage à Henry Corbin - La pensée d'Henry Corbin et le temple maçonnique ", dans Temple et contemplation, Paris, Entrelacs, 2006, p. 9-21.

42. Terme utilisé fréquemment par Durand dès ses premières œuvres. Gilbert Durand, «Psychanalyse de la neige », dans Mercure de France, vol. 318, mai-août 1953, p. 618.

43. Gilbert Durand, «Tâches de l'Esprit et impératif de l'Être », dans Eranos-Jahrbuch, vol.XXXIV/1965, 1966, p. 303-360.

44. Cette expression est influencée par Nicolas Berdiaev. Henry Corbin, En islam iranien, vol. 1, p. 30.

45. Voir par exemple Henry Corbin, « Note sur Existence et Foi », dans Hic et Nunc, n 6, juillet 1933, p. 51-57.

46. Henry Corbin, Histoire de la philosophie islamique, p. 242-246. Les références proposées dans la bibliographie en fin de volume n'aident en rien à faire la lumière sur la justesse des interprétations corbiniennes. Outre le fait qu'il y cite Gilson, Pinès et lui-même, l'hypothèse et l'interprétation corbinienne n'apparaît pas appuyée dans la littérature. Il y aurait à vérifier à quel point cette bibliographie est orientée en faveur de la justification de la " philosophie orientale » d'Avicenne. Une chose est certaine, à la lecture ces pages, il n'y a pas de doute que dans l'esprit corbinien il y a une «philosophie orientale ». À cet égard, et en opposition aux idées de Corbin, voir les travaux d'Anne-Marie Goichon sur le Récit d'Avicenne qui ne sont justement pas cités.

47. Ibid., p. 242.

48. Ibid., p. 343.

49. Henry Corbin, Avicenne et le récit visionnaire, p. 32.

50. « Parler de la dimension polaire comme dimension transcendante de l'individualité terrestre, c'est indiquer que celle-ci comporte une contrepartie, un "partenaire" céleste, et que sa structure totale est celle d'une bi-unité, un unus-ambo. C'est entendre alternativement cet unus-ambo à la $1^{\text {re }}$ et à la 
$2^{\mathrm{e}}$ personne, comme formant une unité dialogique et dialoguante dans l'identité de leur essence et pourtant sans confusion des personnes. C'est pourquoi la dimension polaire s'annonce sous les traits d'une Figure dont les manifestations récurrentes correspondent chaque fois à une expérience absolument personnelle du Spirituel et à la réalisation de cette bi-unité. C'est ainsi que dans l'Iran du $\mathrm{XII}^{\mathrm{e}}$ siècle $\left(\mathrm{VI}^{\mathrm{e}} \mathrm{s}\right.$. de l'hégire) cette Figure reparaît en des contextes qui diffèrent, mais qui ont tous en commun de ressortir à une métaphysique ou à une expérience mystique de la Lumière. » Henry Corbin, L'homme de lumière dans le soufisme iranien, p. 21-22.

51. Daniel Proulx, « La figure de la sophia chez Henry Corbin », dans Femme, Erôs et Philosophie, sous la direction de Elen-Dania Diotte-Besnou, Daniel Proulx et Jean-Michel Counet, Louvain-la-Neuve, EME, 2016, p. 289-317.

52. Voir Henry Corbin, Avicenne et le récit visionnaire.

53. Voir notamment Shihāboddīn Yahyyā Sohravardī, L'archange empourpré, quinze traités et récits mystiques, éd. et trad. de l'arabe et du persan, intro. et notes de Henry Corbin, Paris, Fayard, 1976, 549 p.

54. Gilbert Durand, L’imagination symbolique, 5éd., Paris, P.U.F., 2008 [1964], p. 13.

55. Henry Corbin, L'imagination créatrice, p. 35-36.

56. Sur ce thème, voir Daniel Proulx, « La perception auditive comme perception spirituelle chez Henry Corbin ", dans Quaderni di Studi Indo-mediterranei - Oikosophia, sous la direction de Daniela Boccassini, Milan, Mimesis, 2017, p. 227-246.

57. Jean-Jacques Wunenburger, La raison contradictoire, Paris, Albin Michel, 1990.

58. Sur la distinction entre "méthode » et « approche », voir Daniel Proulx, « Les racines "imaginaires" de la transdisciplinarité », dans L'imaginaire durandien - Enracinements et envols en Terre d'Amérique, sous la direction de Raymond Laprée et Christian Bellehumeur, Québec, Presses de l'Université Laval, 2013, p. 174.

59. Henry Corbin, En islam iranien, vol. 1, p. XIX.

60. Ibid., p. XXVII.

61. Daniel Proulx, «La perception auditive comme perception spirituelle chez Henry Corbin », dans Quaderni di Studi Indo-mediterranei - Oikosophia, p. 227-246.

62. Henry Corbin, En islam iranien, vol. 1, p. 148.

63. Jean-Jacques Wunenburger, La raison contradictoire, p. 13. 